\title{
Simple functional performance tests and mortality in COPD
}

\author{
Milo A. Puhan ${ }^{1,2}$, Lara Siebeling ${ }^{3}$, Marco Zoller $^{4}$, Patrick Muggensturm ${ }^{5}$ and \\ Gerben ter Riet ${ }^{3}$
}

Affiliations: 'Institute for Social and Preventive Medicine, University of Zurich, Zurich, ${ }^{4}$ Dept of General Practice, University of Zurich, Zurich, and ${ }^{5}$ Horten Centre for Patient-Oriented Research and Knowledge Transfer, University of Zurich, Zurich, Switzerland. ${ }^{2}$ Johns Hopkins Bloomberg School of Public Health, Dept of Epidemiology, Baltimore, MD, USA. ${ }^{3}$ Dept of General Practice, University of Amsterdam, Amsterdam, The Netherlands.

Correspondence: M.A. Puhan, Institute for Social and Preventive Medicine, Room HRS G29, Hirshengrabem 84 , $\mathrm{CH}-8001$ Zurich, Switzerland. E-mail: milo.puhandifspm.uzh.ch

ABSTRACT Exercise tests are important to characterise chronic obstructive pulmonary disease patients and predict their prognosis, but are often not available outside of rehabilitation or research settings. Our aim was to assess the predictive performance of the sit-to-stand and handgrip strength tests.

The prospective cohort study in Dutch and Swiss primary care settings included a broad spectrum of patients ( $n=409$ ) with Global Initiative for Chronic Obstructive Lung Disease stages II to IV. To assess the association of the tests with outcomes, we used Cox proportional hazards (mortality), negative binomial (centrally adjudicated exacerbations) and mixed linear regression models (longitudinal health-related quality of life) while adjusting for age, sex and severity of disease.

The sit-to-stand test was strongly (adjusted hazard ratio per five more repetitions of $0.58,95 \% \mathrm{CI} 0.40-0.85$; $\mathrm{p}=0.004)$ and the handgrip strength test moderately strongly $(0.84,95 \%$ CI $0.72-1.00 ; \mathrm{p}=0.04)$ associated with mortality. Both tests were also significantly associated with health-related quality of life but not with exacerbations. The sit-to-stand test alone was a stronger predictor of 2-year mortality (area under curve 0.78 ) than body mass index (0.52), forced expiratory volume in $1 \mathrm{~s}(0.61)$, dyspnoea $(0.63)$ and handgrip strength $(0.62)$.

The sit-to-stand test may close an important gap in the evaluation of exercise capacity and prognosis of chronic obstructive pulmonary disease patients across practice settings.

@ERSpublications

The 1-min sit-to-stand test predicts mortality in COPD patients and can easily be implemented across practice settings http://ow.ly/mxrPx

Received: Aug 222012 | Accepted after revision: Dec 282012 | First published online: March 212013

Clinical trial: This study is registered at www.clinicaltrials.gov with identifier number NCT00706602.

Support statement: Funding was provided by the Swiss National Science Foundation (grant number 3233B0/115216/1), the Dutch Asthma Foundation (grant number 3.4.07.045) and the Zurich Lung League (unrestricted grant).

Conflict of interest: None declared.

Copyright CERS 2013. ERJ Open articles are open access and distributed under the terms of the Creative Commons Attribution Non-Commercial Licence 3.0.

This article was modified in April 2016 to correct errors in the licence information. 


\section{Introduction}

Numerous studies have shown strong associations of exercise capacity with mortality, exacerbations and health-related quality of life (HRQoL) in patients with chronic obstructive pulmonary disease (COPD) [1-5]. In fact, exercise capacity is among the strongest predictors of mortality and showed consistently stronger associations than lung function or dyspnoea $[2,4]$. The body mass index, airflow obstruction, dyspnoea, exercise capacity (BODE) index includes exercise capacity to predict mortality [3]. Multivariable indices inform patients about their prognosis and help them understand what the diagnosis of COPD actually means for them. Also, predictors of outcomes such as exercise capacity help to estimate the risk of future outcomes, the absolute effects of treatments for individuals and, thereby, the benefits and harms of treatments (e.g. number-needed-to-treat). Therefore, assessment of prognosis is of great importance, not only for patients but also for policy makers, regulatory agencies and clinical guideline developers.

Unfortunately, measurement of exercise capacity has not been implemented in most clinical practice settings relevant to COPD patients. Exercise capacity has probably rarely been tested in the vast majority of COPD patients despite its great importance. While the 6-min walk test (6MWT), or the constant workload or incremental exercise tests are commonly used in pulmonary rehabilitation settings, they are not often used in inpatient, outpatient specialist or primary care settings. An important barrier is that the established $6 \mathrm{MWT}$ and constant or incremental workload tests require trained staff, space and equipment [6], which is not commonly available in many practice settings [7].

Over the last 10 years, a significant amount of research has been performed to explore simpler tests, such as the sit-to-stand (STS) or step tests, to measure exercise capacity, or to explore the handgrip strength test to measure upper limb strength. The STS test, where the number of repetitions during either $30 \mathrm{~s}$ or $1 \mathrm{~min}$ are counted, yields reproducible results in elderly people (intraclass correlation coefficients $\geqslant 0.84$ ) and the STS test showed high correlations with established tests for exercise capacity such as 6-min walking distance (6MWD) $(r>0.7)$ [8-10]. The STS and 6MWTs showed very similar correlations with other validation measures, indicating that they may measure similar aspects of functional exercise capacity [8].

However, we were unable to find studies that directly associated simple exercise tests with clinical outcomes. If STS or handgrip strength tests are to be used in practice to assess health status and associated prognosis, or are to be implemented in prognostic indices, a validation of their predictive properties is needed. Such a direct validation provides stronger evidence for predictive properties than studies, which show high correlations of STS with commonly used exercise tests and indirectly suggests a link of simple exercise tests to clinical outcomes. Therefore, our aim was to assess whether and, if so, how strongly the STS and handgrip strength tests are associated with mortality, exacerbations and longitudinal HRQoL in a diverse population of COPD patients.

\section{Methods \\ Study design and population}

We based our analyses on a prospective multicentre cohort study with COPD patients from primary care in Switzerland and the Netherlands [11, 12]. At inclusion (April 2008 to August 2009), all patients (aged $\geqslant 40$ years) had Global Initiative for Chronic Obstructive Lung Disease (GOLD) stages II-IV and had been free of exacerbations for $\geqslant 4$ weeks. The only exclusion criteria were life expectancy of $<12$ months, dementia or psychotic morbidity. All included patients provided written informed consent. The study has been approved of by all local ethics committees and is registered at www.clinicaltrials.gov (identifier number NCT00706602).

\section{1-min STS test and handgrip strength test}

Using the STS test protocol [10], trained study nurses asked patients to sit down on a chair (height 46-48 cm) without arm rests, keep their legs apart with about 90 degrees knee flexion and aligned with their hips, and to hold their hands stationary on their hips. Patients were asked to stand up and to sit down once or twice in order to familiarise them with the task and to assess its feasibility and safety. Study nurses instructed patients about the duration of the test $(1 \mathrm{~min})$ and to do as many repetitions as possible at a selfpaced speed allowing for short breaks if needed but without using the arms for support. Study nurses started the test by giving the command "attention, ready, go". When $15 \mathrm{~s}$ were left patients were told "You have 15 seconds left until the test is over".

To measure handgrip strength we used the Jamar Hydraulic Hand Dynamometer (JA Preston Corporation, Jackson, MI, USA), which is widely used and serves as reference for evaluation of other devices [13]. Patients were seated with the shoulders adducted, elbows flexed to $90^{\circ}$ and forearms in a neutral position. Trained study nurses then instructed patients to squeeze the handle as much as possible and read to the 
nearest kilogramme where the needle stopped. As recommended, we used the best of the six measurements (three times for each hand) for the statistical analyses [13].

\section{Outcome measurements}

We focused on three patient-important outcomes, namely, mortality, exacerbations and COPD-specific HRQoL. Deaths were captured at the biannual follow-up assessments (at 6, 12, 18 and 24 months) and exact dates of death were ascertained from the treating primary care doctors. We focused on all-cause death since determination of causes of death is prone to misclassification and is difficult to perform, even if centrally adjudicated [14].

Potential exacerbations were captured through patient interviews (biannual assessments) and through review of all patient records 2 years after enrolment. In both Switzerland and the Netherlands, primary care doctors play a central role in coordinating patient care and reports from other healthcare providers (e.g. hospitals and pulmonologists) are sent to the primary care doctors. These reports, as well as the primary care doctors' case records, were available to us. All potential exacerbations were centrally adjudicated by committees that comprised three independent experts (general practitioners with special expertise in pulmonary medicine and pulmonologists) in Switzerland and four in the Netherlands. The experts first independently reviewed the documents and determined the number and dates of exacerbations for each patient. We used an event-based definition for exacerbations with two criteria that had to be fulfilled. 1) Unscheduled physician contact in a hospital, private practice or by telephone for worsening of dyspnoea, cough, increased sputum production or a change in sputum colour; and 2) electronic or handwritten documentation of new prescription or a dosage increase of systemic steroids or new prescription of an antibiotic. The experts sent their reviews to the Swiss and Dutch study coordinating centres. At the consensus meetings of the two adjudication committees, the members compared their independent reviews on those patients for whom discordances existed and reached a consensus on the number and dates of exacerbations. More details about the adjudication process will be reported elsewhere.

To assess COPD-specific HRQoL, we used the validated German and Dutch self-administered, standardised versions of the Chronic Respiratory Questionnaire (CRQ). The CRQ provides scores in four different domains (dyspnoea, fatigue, emotional and mastery), each on a scale of one to seven, where one is the worst score indicating very poor HRQoL and seven the best $[15,16]$. Patients completed the CRQ at baseline and biannually up to the 2-year visit.

\section{Statistical analysis}

We used regression analyses with the outcomes as dependent and the exercise tests as independent variables (separate analysis for each outcome and for STS and handgrip strength tests, respectively). We used Cox proportional hazards models to assess the association between the exercise tests and mortality (up to 2 years). As exacerbations are usually correlated within patients, we used negative binomial regression models to assess the association between the exercise tests and the number of exacerbations (incidence rate ratio). To assess the association between the exercise tests and HRQoL from baseline up to 2 years we used random-effects linear regression models that took into account the within-person (repeated measurements) and between-person variability. We adjusted the analyses for age, sex, baseline dyspnoea, baseline forced expiratory volume in $1 \mathrm{~s}$ (FEV1) \% predicted and the use of long-acting bronchodilators with or without inhaled corticosteroids (at baseline). In sensitivity analyses, we imputed a zero for patients who did not perform the STS test at baseline. Finally, we calculated the area under the curve for a number of commonly used predictors, such as FEV1 \% predicted, age, dyspnoea and body mass index, and their combinations.

We did not have any missing variables for the handgrip strength test or the confounders. Not all patients were able to complete the STS test and we performed sensitivity analyses as described above. We did not perform formal sample size calculations for this analysis, but we have described details for sample size calculations for this cohort study before [11]. We conducted all analyses using Stata for Windows (version 11.2, College Station, TX, USA).

\section{Results}

Study population

Detailed characteristics of the broad study population of 409 COPD patients can be found elsewhere [12]. In brief, at enrolment mean \pm SD age was $67.3 \pm 10.0$ years, $57 \%$ were male, $63.8 \%$ had GOLD stage II, $21.8 \%$ stage III and $14.4 \%$ stage IV, respectively, and mean \pm SD degree of dyspnoea on the Medical Research Council scale was $1.87 \pm 1.46 .38(9.3 \%)$ patients had died within the first 2 years of observation (total observation time of 782 person-years). Only 19 (4.6\%) patients dropped out of the study, because of worsening of physical condition $(n=4)$, overburden $(n=4)$, psychiatric condition $(n=3)$, lost contact $(n=1)$ or other reasons $(n=7) .204(49.9 \%)$ patients had no exacerbation, $101(24.7 \%)$ had one 
exacerbation, $33(8.1 \%)$ had two and $71(17.4 \%)$ patients had three or more exacerbations during the first 2 years of observation. $93.3 \%$ of the adjudicated exacerbations were treated on an outpatient and $6.7 \%$ on an inpatient basis.

STS and handgrip strength test results at baseline

$374(91.4 \%)$ patients were able to perform the STS test at baseline. Reasons for not performing a STS were musculoskeletal problems (osteoarthritis, back pain or recent orthopaedic surgery). The mean \pm SD number of repetitions during the STS test was $18.9 \pm 8.8$. STS test results were lower for females compared with males, for patients with higher GOLD stage, for patients experiencing more dyspnoea and for patients with more comorbidities (table 1). All patients were able to perform the handgrip strength test at baseline. The mean \pm SD maximum handgrip strength was $35.8 \pm 12.8 \mathrm{~kg}$. There was high reproducibility across the three attempts on the right side (means of $33.4,32.9$ and $32.6 \mathrm{~kg}$ on first, second and third attempts, respectively) and left side (means of $30.8,30.7$ and $30.5 \mathrm{~kg}$ ), with intraclass correlation coefficients of 0.88 for both sides. Again, test results were lower for females, and in patients with more dyspnoea and more comorbidities, whereas patients with higher GOLD stages did not have lower upper limb strength. The correlation between the number of repetitions during the STS test and maximal handgrip strength was 0.45 .

\section{Association of the STS test with mortality, exacerbations and HRQoL}

The STS test at baseline was substantially lower in patients who died subsequently than in patients who were alive at 2 years (11.8 versus 19.5 repetitions; table 2). We found a strong association of the STS test with mortality. The adjusted analysis showed a hazard ratio of 0.90 per one more repetition ( $95 \%$ CI $0.83-0.97$; $\mathrm{p}=0.004)$, equivalent to a hazard ratio of $0.58(95 \%$ CI $0.40-0.85 ; \mathrm{p}=0.004)$ per five more repetitions (table 2).

We did not find an association of the STS test with exacerbations (incidence rate ratio per 1 more repetition close to 1.0), but we did find statistically significant associations of the STS test with all four domains of the CRQ, as assessed over 24 months. The adjusted effect of five more repetitions during the STS test was 0.26 (95\% CI 0.19-0.34) for the dyspnoea, 0.19 (95\% CI 0.13-0.26) for the fatigue, 0.10 (95\% CI 0.04-0.16) for the emotional function and 0.08 (95\% CI 0.02-0.13) for the mastery domain. Six patients completed only four repetitions during the STS test and two patients three and one repetitions, respectively. Excluding these patients from the analyses led to almost identical estimates, as in the main analyses. Finally, in additional sensitivity analyses, we imputed a zero for those patients who were unable to perform the STS test at baseline $(n=35)$. The analysis for the complete set of patients $(n=409)$ showed almost identical results to those in the main analysis.

TABLE 1 Sit-to-stand (STS) and handgrip strength tests at baseline

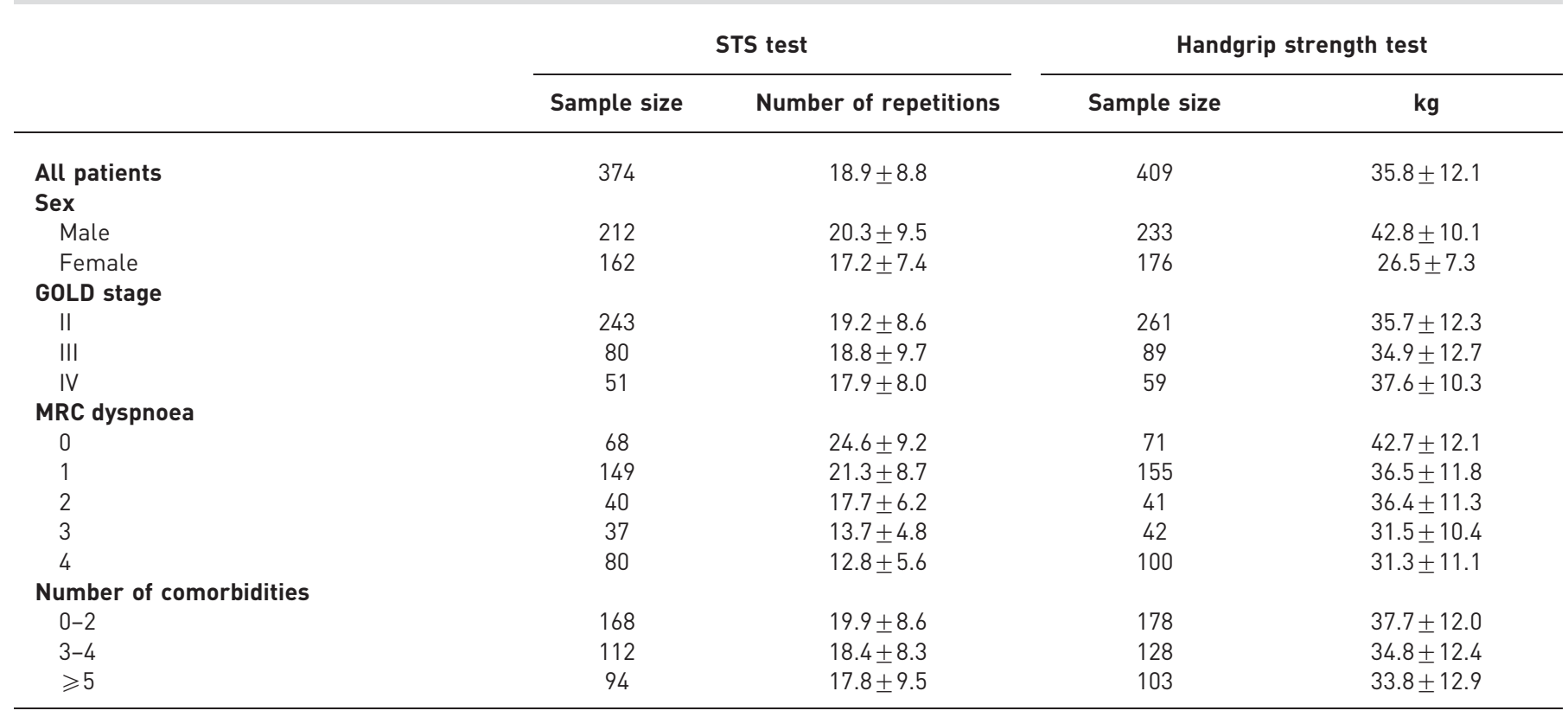

Data are presented as n or mean \pm SD. GOLD: Global Initiative for Chronic Obstructive Lung Disease; MRC: Medical Research Council. 
TABLE 2 Association of the sit-to-stand (STS) test with mortality, exacerbations and health-related quality of life (HRQoL)

Mortality

\begin{tabular}{|c|c|c|}
\hline & \multicolumn{2}{|c|}{ Mortality } \\
\hline & Patients alive at 2 years & Patients dead at 2 years \\
\hline \multirow[t]{3}{*}{$\begin{array}{l}\text { STS test number of repetitions in } 1 \mathrm{~min} \\
\text { Association of STS with mortality }\end{array}$} & $\begin{array}{l}\qquad 19.5 \pm 8.7 \\
\text { Unadjusted association (hazard ratio per } 1 \text { more repetition) } \\
\text { Adjusted association (hazard ratio per } 1 \text { more repetition) } \\
\text { Adjusted association (hazard ratio per } 5 \text { more repetitions) } \\
\text { Covariates }\end{array}$ & $\begin{array}{c}11.8 \pm 6.3 \\
0.86(95 \% \mathrm{Cl} 0.81-0.92), p<0.0001 ; z=-4.73 \\
0.90(95 \% \mathrm{Cl} 0.83-0.97), p=0.004 ; z=-2.84 \\
0.58(95 \% \mathrm{Cl} 0.40-0.85) p=0.004 ; z=-2.84 \\
\text { Age 1.08 (SE 0.026, } z=3.17, p=0.002) \\
\text { FEV1 0.98 (SE 0.012, } z=-1.76, p=0.078 \text { ); } \\
\text { dyspnoea 1.12 (SE } 0.185, z=0.69, p=0.49 \text { ); } \\
\text { LABA/ICS 0.56 (SE } 0.29, z=-1.11, p=0.269 \text { ) }\end{array}$ \\
\hline & \multicolumn{2}{|c|}{ Exacerbations } \\
\hline & Patients with no exacerbation during 2 years of follow-up & Patients with $\geqslant 1$ exacerbation during 2 years of follow-up \\
\hline $\begin{array}{l}\text { STS test number of repetitions in } 1 \mathrm{~min} \\
\text { Association of STS test with exacerbations }\end{array}$ & $\begin{array}{l}19.4 \pm 8.5 \\
\text { Unadjusted association (incidence rate ratio per } 1 \text { more repetition) } \\
\text { Adjusted association (incidence rate ratio per } 1 \text { more repetition) } \\
\text { Covariates } \\
\text { HRQoL from baselir }\end{array}$ & $\begin{array}{c}18.1 \pm 8.1 \\
0.99(95 \% \mathrm{Cl} 0.97-1.01), p=0.21 ; z=-1.26 \\
1.00(95 \% \mathrm{Cl} 0.98-1.02), p=0.94 ; z=0.08 \\
\text { Age 1.00 (SE 0.007, } z=-0.48, p=0.63) \\
\text { FEV1 } 0.98 \text { (SE 0.005, } z=-5.02, p<0.001) \\
\text { dyspnoea 1.06 (SE 0.067, } z=0.89, p=0.38 \text { ); } \\
\text { LABA/ICS 1.52 (SE 0.34, } z=1.87, p=0.062) \\
\text { 2-year follow-up }\end{array}$ \\
\hline Association of STS test with CRQ dyspnoea & $\begin{array}{c}\text { Unadjusted difference on scale from } 1-7 \text { per } 1 \text { more repetition } \\
\text { Adjusted difference on scale from 1-7 per } 1 \text { more repetition } \\
\text { Adjusted difference on scale from 1-7 per } 5 \text { more repetitions } \\
\text { Covariates }\end{array}$ & $\begin{array}{c}0.06(95 \% \mathrm{Cl} 0.04-0.07), p<0.001 ; z=7.55 \\
0.05(95 \% \mathrm{Cl} 0.04-0.07), p<0.001 ; z=7.10 \\
0.26(95 \% \mathrm{Cl} 0.19-0.34), p<0.001 ; z=7.10 \\
\text { Age } 0.017 \text { (SE } 0.007, z=2.54, p=0.011 \text { ); } \\
\text { FEV1 0.026 (SE 0.004, } z=-6.48, p<0.001 \text { ) } \\
\text { LABA/ICS - } 0.396 \text { (SE } 0.16, z=-2.41, p=0.016 \text { ) }\end{array}$ \\
\hline Association of STS test with CRQ fatigue & $\begin{array}{c}\text { Unadjusted difference on scale from 1-7 per } 1 \text { more repetition } \\
\text { Adjusted difference on scale from 1-7 per } 1 \text { more repetition } \\
\text { Adjusted difference on scale from 1-7 per } 5 \text { more repetitions } \\
\text { Covariates }\end{array}$ & $\begin{array}{l}0.06 \text { (95\% Cl 0.04-0.07), } p<0.001 ; z=8.57 \\
0.04 \text { (95\% Cl } 0.03-0.05), p<0.001 ; z=6.06 \\
0.19(95 \% \mathrm{Cl} 0.13-0.26), p<0.001 ; z=6.06 \\
\text { Age } 0.007 \text { (SE 0.005, } z=1.34, p=0.18 \text { ); } \\
\text { FEV1 -0.007 (SE 0.005, } z=-2.04, p=0.042 \text { ); } \\
\text { dyspnoea } 0.361 \text { (SE } 0.038, z=9.51, p<0.001 \text { ); } \\
\text { LABA/ICS } 0.108 \text { (SE } 0.13, z=0.81, p=0.42 \text { ) }\end{array}$ \\
\hline $\begin{array}{l}\text { Association of STS test with CRQ emotional } \\
\text { function }\end{array}$ & $\begin{array}{c}\text { Unadjusted difference on scale from 1-7 per } 1 \text { more repetition } \\
\text { Adjusted difference on scale from 1-7 per } 1 \text { more repetition } \\
\text { Adjusted difference on scale from } 1-7 \text { per } 5 \text { more repetitions } \\
\text { Covariates }\end{array}$ & $\begin{array}{l}0.03(95 \% \mathrm{Cl} 0.02-0.05), p<0.001 ; z=5.33 \\
0.02(95 \% \mathrm{Cl} 0.01-0.03), p=0.001 ; z=3.24 \\
0.10(95 \% \mathrm{Cl} 0.04-0.16), p=0.001 ; z=3.24 \\
\text { Age } 0.013 \text { (SE 0.005, } z=2.52, p=0.012 \text { ) } \\
\text { FEV1 -0.009 (SE 0.003, } z=-2.81, p=0.005 \text { ); } \\
\text { dyspnoea } 0.357 \text { (SE } 0.036, z=9.87, p<0.001 \text { ); } \\
\text { LABA/ICS } 0.169 \text { (SE } 0.13, z=1.32, p=0.19 \text { ) }\end{array}$ \\
\hline Association of STS test with CRQ mastery & $\begin{array}{c}\text { Unadjusted difference on scale from 1-7 per } 1 \text { more repetition } \\
\text { Adjusted difference on scale from 1-7 per } 1 \text { more repetition } \\
\text { Adjusted difference on scale from 1-7 per } 5 \text { more repetitions } \\
\text { Covariates }\end{array}$ & $\begin{array}{c}0.03(95 \% \mathrm{Cl} 0.02-0.04), p<0.001 ; z=5.79 \\
0.02(95 \% \mathrm{Cl} 0.00-0.03), p=0.005 ; z=2.84 \\
0.08(95 \% \mathrm{Cl} 0.02-0.13), p=0.005 ; z=2.84 \\
\text { Age 0.008 (SE 0.005, } z=1.69, p=0.09 \text { ); } \\
\text { FEV1 - } 0.002 \text { (SE 0.003, } z=-0.65, p=0.51 \text { ); } \\
\text { dyspnoea } 0.340 \text { (SE 0.033, } z=10.41, p<0.001 \text { ); } \\
\text { LABA/ICS }-0.053 \text { (SE } 0.116, z=-0.46, p=0.64 \text { ) }\end{array}$ \\
\hline
\end{tabular}

Data are presented as mean \pm SD, unless otherwise stated. FEV1: forced expiratory volume in $1 \mathrm{~s}$; LABA: long-acting $\beta$-agonist; ICS: inhaled corticosteroid; CRQ; Chronic Respiratory Questionnaire. $z$-values are reported to show the relative strength of association of each variable in the multivariable regression models. All regression coefficients for continuous variables except for handgrip strength are per increase of 1 unit 11 year for age, 1\% predicted for FEV1 and 1 point for dyspnoeal.

Association of the handgrip strength with mortality, exacerbations and HRQoL

Handgrip strength at baseline was substantially lower in patients who died subsequently than in patients who were alive at 2 years $(30.1 \mathrm{~kg}$ versus $36.4 \mathrm{~kg}$ ) (table 3). We also found a statistically significant association of handgrip strength with mortality. The adjusted analysis showed a hazard ratio of 0.97 per one more kilogramme $(95 \%$ CI $0.94-0.99 ; \mathrm{p}=0.04)$ equivalent to a hazard ratio of 0.84 (95\% CI $0.72-1.00$; $\mathrm{p}=0.04)$ per five more kilogrammes (table 3 ).

As for the STS test, we did not find an association of handgrip strength with exacerbations. Handgrip strength was significantly associated with three CRQ domains but not with mastery. As for the STS test, we found the strongest association of handgrip strength with dyspnoea (adjusted effect of five more kilogrammes $0.14,95 \%$ CI $0.09-0.20)$, followed by fatigue $(0.12,95 \%$ CI $0.08-0.17)$, emotional function (0.06, 95\% CI 0.02-0.10) and mastery (0.01, 95\% CI -0.03-0.05).

Comparison of prediction of mortality of STS and handgrip strength test with established predictors The age, dyspnoea and airflow obstruction (ADO) index, the STS test and combinations of both indices with the STS test were strong predictors of 2-year mortality with areas under the curve of around 0.8 (fig. 1). The STS test alone predicted 2-year mortality almost as well as the ADO index. Handgrip strength and the BODE index without an exercise test were both much weaker predictors of 2-year mortality, but a 
TABLE 3 Association of handgrip strength with mortality, exacerbations and health-related quality of life (HRQoL)

Mortality

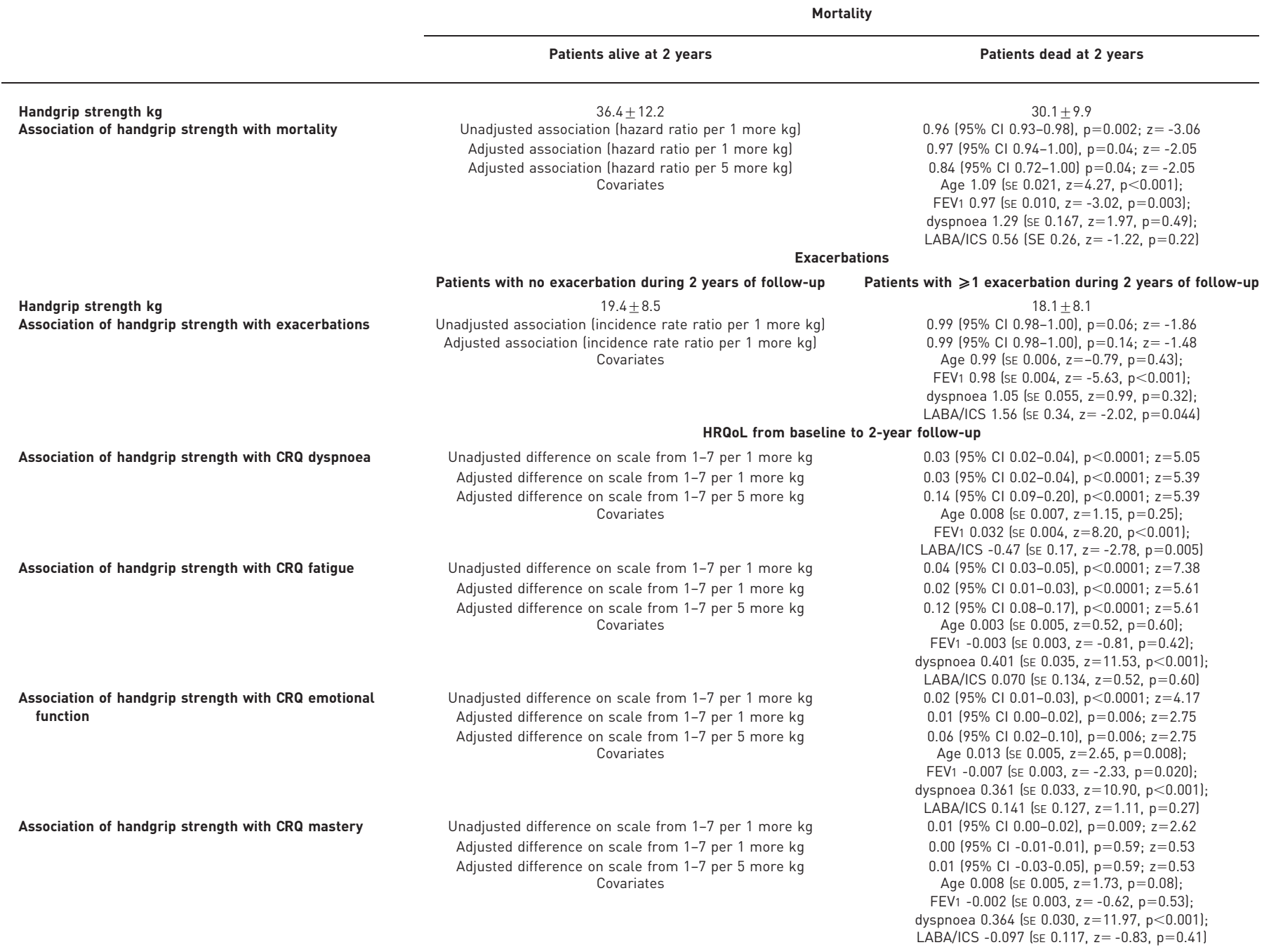

Data are presented as mean \pm SD, unless otherwise stated. FEV1: forced expiratory volume in 1 s; LABA: long-acting $\beta$-agonist; ICS: inhaled corticosteroid; CRQ; Chronic Respiratory Questionnaire.

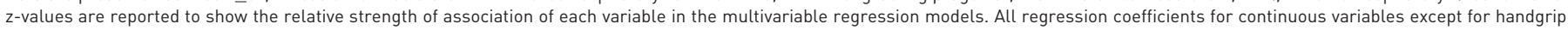
strength are per increase of 1 unit (1 year for age, 1\% predicted for FEV 1 and 1 point for dyspnoea).

BODE index with the STS test instead of the 6MWD showed excellent prediction of 2-year mortality. Age alone was also a good predictor, whereas all other predictors included in the BODE or ADO indices were either poor or moderate predictors when used alone.

\section{Discussion}

We found that upper limb strength measured by the handgrip test and, in particular, the 1-min STS test as a measure of exercise capacity are strongly and independently associated with mortality and HRQoL over 24 months of observation, but we did not find significant associations with exacerbations. The STS test alone is as good a predictor of mortality as the ADO index and may replace BODE's 6MWD if the latter is not available.

Tables 1 and 2, where we reported the full regression models, indicate that the STS test was a stronger predictor of mortality than FEV1 \% predicted, dyspnoea or the use of inhaled drugs. The strong predictive properties are also reflected by the area under the curve of 0.78 (fig. 1). Earlier studies using the 6MWT or incremental exercise test show similar results $[1,2,5,17]$. Upper limb strength was less strongly, but still statistically significantly associated with mortality, but the area under the curve (0.62) suggests it may be of limited use to predict mortality in COPD patients. Although direct comparisons of the predictive properties 
Predictors of 2-year mortality

\begin{tabular}{|c|c|c|}
\hline ADO + STS test & $\rightarrow$ & $0.82(0.74-0.90)$ \\
\hline$A D O$ + handgrip strength & $\longrightarrow$ & $0.80(0.71-0.89)$ \\
\hline ADO & $\longrightarrow$ & $0.80(0.71-0.89)$ \\
\hline BODE (with STS test) & $\rightarrow$ & $0.78(0.70-0.87)$ \\
\hline STS test & $\longrightarrow$ & $0.78(0.69-0.86)$ \\
\hline Age years & $\longrightarrow$ & $0.75(0.65-0.84)$ \\
\hline BODE (with handgrip strength) & $\longrightarrow$ & $0.68(0.57-0.80)$ \\
\hline BOD (without exercise capacity) & $\longrightarrow$ & $0.66(0.55-0.77)$ \\
\hline Dyspnoea (MRC) & $\longrightarrow$ & $0.63(0.52-0.72)$ \\
\hline Handgrip strength & $\longrightarrow$ & $0.62(0.52-0.72)$ \\
\hline FEV $1 \%$ predicted & - & $0.61(0.51-0.72)$ \\
\hline $\mathrm{BMI} \mathrm{kg} \cdot \mathrm{m}^{-2}$ & - & $0.52(0.41-0.63)$ \\
\hline
\end{tabular}

Area under the curve

(95\% CI)

FIGURE 1 Comparison of predictors to predict 2-year mortality in chronic obstructive pulmonary disease patients. The figure shows the area under the curve (with 95\% confidence interval) for each predictor and for combinations of predictors. An area under the curve of 0.5 indicates prediction no better than chance and 1.0 perfect prediction. ADO: age, dyspnoea and airflow obstruction; STS: sit-to-stand; BMI: body mass index; BODE: BMI, airflow obstruction, dyspnoea, exercise capacity; MRC: Medical Research Council; FEV1: forced expiratory volume in $1 \mathrm{~s}$.

of exercise tests are scarce, the current body of evidence suggests that the simpler STS test and the 6MWT or incremental exercise test can be used to predict outcomes in COPD.

In most practice settings, exercise tests are uncommonly performed in COPD patients because these tests require equipment, space and trained staff. In addition, reimbursement for the 6MWT is not always guaranteed. We believe that the STS test offers an attractive alternative. The STS test, which, according to our protocol, lasts $1 \mathrm{~min}$ and appears to measure similar aspects of exercise capacity to the $6 \mathrm{MWT}[8,9]$. It may also be responsive to change [18]. Its simplicity makes it an ideal test for primary but also for acute care settings [19]. The handgrip strength test requires a handgrip dynamometer (one time investment of about $\$ 300$ ), but it is very simple to perform and most, and in our study, all, patients are able to perform the test. However, we think that this test alone is insufficient to inform patients and physicians about exercise capacity because it does not test large or multiple muscles as do other tests, which is probably the reason for not being a strong predictor of prognosis. Handgrip strength might, however, serve as a first test to identify patients with low upper limb strength who could then undergo more extensive testing of exercise capacity.

Some questions about the usefulness of the STS and handgrip strength tests remain. We did not find a significant association of the two tests with exacerbations. A possible reason for this is that patients in our cohort rarely experienced severe exacerbations requiring hospital admission. Given the strong association of the tests with mortality it is possible that, in patients with more severe COPD who suffer from severe exacerbations, a significant association may exist. In the present analyses, the number of hospital admissions was too low to restrict the analysis to severe exacerbations, but as soon as more years of follow-up are available this or other studies should assess the association of the STS and handgrip strength tests with severe exacerbations. Additional studies may also directly compare the predictive properties of the STS and 6MWT and explore whether the STS would be able to contribute as much to the BODE index as the $6 \mathrm{MWD}$. Finally, the goal of our study was to assess the predictive properties of the STS and handgrip strength tests, but we did not evaluate their properties when used as outcome measures. These simple tests may be used in randomised trials as additional outcome measures. However, before tests like the STS or handgrip strength test are routinely used in trials, their responsiveness to change and minimal important difference need to be established, as has been done for the 6MWD [20].

Strengths of this study include a carefully developed study protocol [11] that was successfully implemented, as illustrated by the completeness of data collection and very low number of dropouts. The large and broad cohort from primary care is another strength and we think that our results are applicable to other populations because our cohort is likely to reflect a typical primary care COPD population. A limitation of this study is that we did not directly compare the predictive properties of the STS and handgrip strength test with those of the 6MWT or other tests. We restricted our study to measurements that are feasible in a primary care setting. Another limitation is that we did not ascertain mild exacerbations, which we defined as worsening of symptoms that did not lead to the prescription of antibiotics or systemic corticosteroids. At the design stage of this cohort study we decided to focus on moderate-to-severe exacerbations because of their great impact on the patients' HRQoL and prognosis and because a central adjudication would be very challenging for mild exacerbations. 
In conclusion, in this prospective cohort study of primary care COPD patients, we found that the STS test as a measure of exercise capacity is a strong predictor of mortality and HRQoL and may be an attractive option with which to assess exercise capacity in COPD patients in both clinical practice and research. The availability of such a simple test may close an important gap in the evaluation of the prognosis of COPD patients across different settings.

\section{Acknowledgements}

We thank U. Schafroth (Horten Centre for Patient-oriented Research, University of Zurich, Zurich, Switzerland) and A. Karsten (Academic Medical Centre, Department of General Practice, University of Amsterdam, Amsterdam, the Netherlands) and the participating general practitioners and COPD patients in Switzerland and the Netherlands (Stichting Gezondheidscentra Amsterdam Zuidoost, Amsterdam, and Zorggroep Almere, Amersfort, the Netherlands) who made this study possible by their enthusiastic involvement.

\section{References}

1 Pinto-Plata VM, Cote C, Cabral H, et al. The 6-min walk distance: change over time and value as a predictor of survival in severe COPD. Eur Respir J 2004; 23: 28-33.

2 Marquis K, Debigare R, Lacasse Y, et al. Midthigh muscle cross-sectional area is a better predictor of mortality than body mass index in patients with chronic obstructive pulmonary disease. Am J Respir Crit Care Med 2002; 166: 809-813.

3 Celli BR, Cote CG, Marin JM, et al. The body-mass index, airflow obstruction, dyspnea, and exercise capacity index in chronic obstructive pulmonary disease. N Engl J Med 2004; 350: 1005-1012.

4 Puhan MA, Garcia-Aymerich J, Frey M, et al. Expansion of the prognostic assessment of patients with chronic obstructive pulmonary disease: the updated BODE index and the ADO index. Lancet 2009; 374: 704-711.

5 Spruit MA, Polkey MI, Celli B, et al. Predicting outcomes from 6-minute walk distance in chronic obstructive pulmonary disease. J Am Med Dir Assoc 2012; 13: 291-297.

6 American Thoracic Society. ATS statement: guidelines for the six-minute walk test. Am J Respir Crit Care Med 2002; 166: 111-117.

Puhan MA, Zoller M, ter Riet G. COPD: more than respiratory. Lancet 2008; 371: 27-28. Bohannon RW. Measurement of sit-to-stand among older adults. Top Geriatr Rehabil 2012; 28: 11-16.

Jones CJ, Rikli RE, Beam WC. A 30-s chair-stand test as a measure of lower body strength in community-residing older adults. Res Q Exerc Sport 1999; 70: 113-119.

10 Ozalevli S, Ozden A, Itil O, et al. Comparison of the sit-to-stand test with 6 min walk test in patients with chronic obstructive pulmonary disease. Respir Med 2007; 101: 286-293.

11 Siebeling L, ter Riet G, van der Wal WM, et al. ICE COLD ERIC - international collaborative effort on chronic obstructive lung disease: exacerbation risk index cohorts - study protocol for an international COPD cohort study. BMC Pulm Med 2009; 9: 15.

12 Siebeling L, Puhan MA, Muggensturm P, et al. Characteristics of Dutch and Swiss primary care COPD patients baseline data of the ICE COLD ERIC study. Clin Epidemiol 2011; 3: 273-283.

13 Roberts HC, Denison HJ, Martin HJ, et al. A review of the measurement of grip strength in clinical and epidemiological studies: towards a standardised approach. Age Ageing 2011; 40: 423-429.

14 McGarvey LP, John M, Anderson JA, et al. Ascertainment of cause-specific mortality in COPD: operations of the TORCH Clinical Endpoint Committee. Thorax 2007; 62: 411-415.

15 Puhan MA, Behnke M, Laschke M, et al. Self-administration and standardisation of the chronic respiratory questionnaire: a randomised trial in three German-speaking countries. Respir Med 2004; 98: 342-350.

16 Wijkstra PJ, TenVergert EM, Van Altena R, et al. Reliability and validity of the chronic respiratory questionnaire (CRQ). Thorax 1994; 49: 465-467.

17 Cote CG, Pinto-Plata V, Kasprzyk K, et al. The 6-min walk distance, peak oxygen uptake, and mortality in COPD. Chest 2007; 132: 1778-1785.

18 Patel M, Canavan J, Clark A, et al. The effect of pulmonary rehabilitation on the sit-to-stand test in COPD. Eur Respir J 2011; 38: Suppl. 55, 226 S.

19 Annweiler C, Schott AM, Abellan van Kan G, et al. The five-times-sit-to-stand test, a marker of global cognitive functioning among community-dwelling older women. J Nutr Health Aging 2011; 15: 271-276.

20 Puhan MA, Chandra D, Mosenifar Z, et al. The minimal important difference of exercise tests in severe COPD. Eur Respir J 2011; 37: 784-790. 